\title{
Septate uterus with complete vaginal agenesis: an undescribed Müllerian malformation
}

\author{
M. L. Marotta • J. Donnez • N. Michaux • M. Dupont • \\ O. Donnez
}

Received: 29 August 2012 / Accepted: 18 October 2012 / Published online: 18 November 2012

(C) Springer-Verlag Berlin Heidelberg 2012

\section{Introduction}

Müllerian malformations are the consequence of failure in the fusion or resorption processes of the two Müllerian ducts at around 9 weeks of gestation [1]. The mean prevalence in the general population is as high as $7 \%$ [2].

Vaginal agenesis is a rare female congenital tract anomaly. Diagnosis is often made in adolescence due to amenorrhea or cyclic abdominal pain, and its incidence is around 1 in 4,000 female births. Mayer-Rokitansky-Küster-Hauser (MRKH) syndrome is the most frequent manifestation, with a vaginal dimple of varying depth $(2-7 \mathrm{~cm})$ and sometimes a rudimentary uterus or horn [3]. In this particular case, the goal of treatment (surgical or nonsurgical) is to allow normal sexual intercourse, but restoring fertility is not possible.

M. L. Marotta $\cdot$ N. Michaux $\cdot$ O. Donnez

CHU Mont-Godinne, Gynecology,

Université Catholique de Louvain,

5530 Yvoir, Belgium

\section{J. Donnez}

Pôle de Recherche en Gynécologie, Institut de Recherche

Expérimentale et Clinique (IREC),

Université Catholique de Louvain (UCL),

1200 Brussels, Belgium

\section{J. Donnez}

Department of Gynecology, Cliniques Universitaires Saint-Luc, 1200 Brussels, Belgium

\section{Dupont}

CHU Mont-Godinne, Radiology,

Université Catholique de Louvain,

5530 Yvoir, Belgium

\section{J. Donnez $(\bowtie)$}

Department of Gynecology, Cliniques Universitaires Saint-Luc, Catholic University of Louvain,

Avenue Hippocrate 10,

1200 Brussels, Belgium

e-mail: jacques.donnez@uclouvain.be
Different approaches have been described, such as Ingram's modification of the Frank technique, the McIndoe procedure, the Vecchietti method, and the Williams vaginoplasty with its Creatsas modification [3]. Here, we report the first case of complete vaginal agenesis associated with a septate uterus to be described in the literature.

\section{Case report}

An 11-year-old girl was referred to our department because of abdominal pain. At the age of 8 months, she had undergone cardiac interventricular communication closure, and at 10 months, surgery for vesicoureteral reflux. She had been suffering from primary amenorrhea and cyclic abdominal pain for 4 months. Abdominal examination was normal. The blood sample was also normal, without any inflammation or anemia.

Gynecological examination showed a normal vulva with a non-perforated intact hymen. The presence of a blind vaginal dimple was suspected.

Pelvic ultrasound showed a uterus measuring $45 \times 20 \mathrm{~mm}$ with hematometra and, on the left side, another myometrial structure measuring $30 \times 26 \mathrm{~mm}$ containing collected blood. Both ovaries were normal.

The axial view of T2-weighted pelvic MRI images revealed a uterus with a uterocervical septum (Fig. 1a, c) and hematometra and hematocervix on both sides (Fig. 1a-c). The cervix was of normal length. No vaginal tissue could be seen.

Diagnostic laparoscopy confirmed the presence of a single uterus with a heart-shaped corpus (Fig. 2a). Endometriotic implants were found in the peritoneum of the uterovesical space and Douglas pouch. Severe adhesions between both ovaries and the ovarian fossa were due to endometriosis (Fig. 2b). Extensive adhesiolysis was performed and peritoneal biopsies were taken, confirming the presence of endometriosis. 

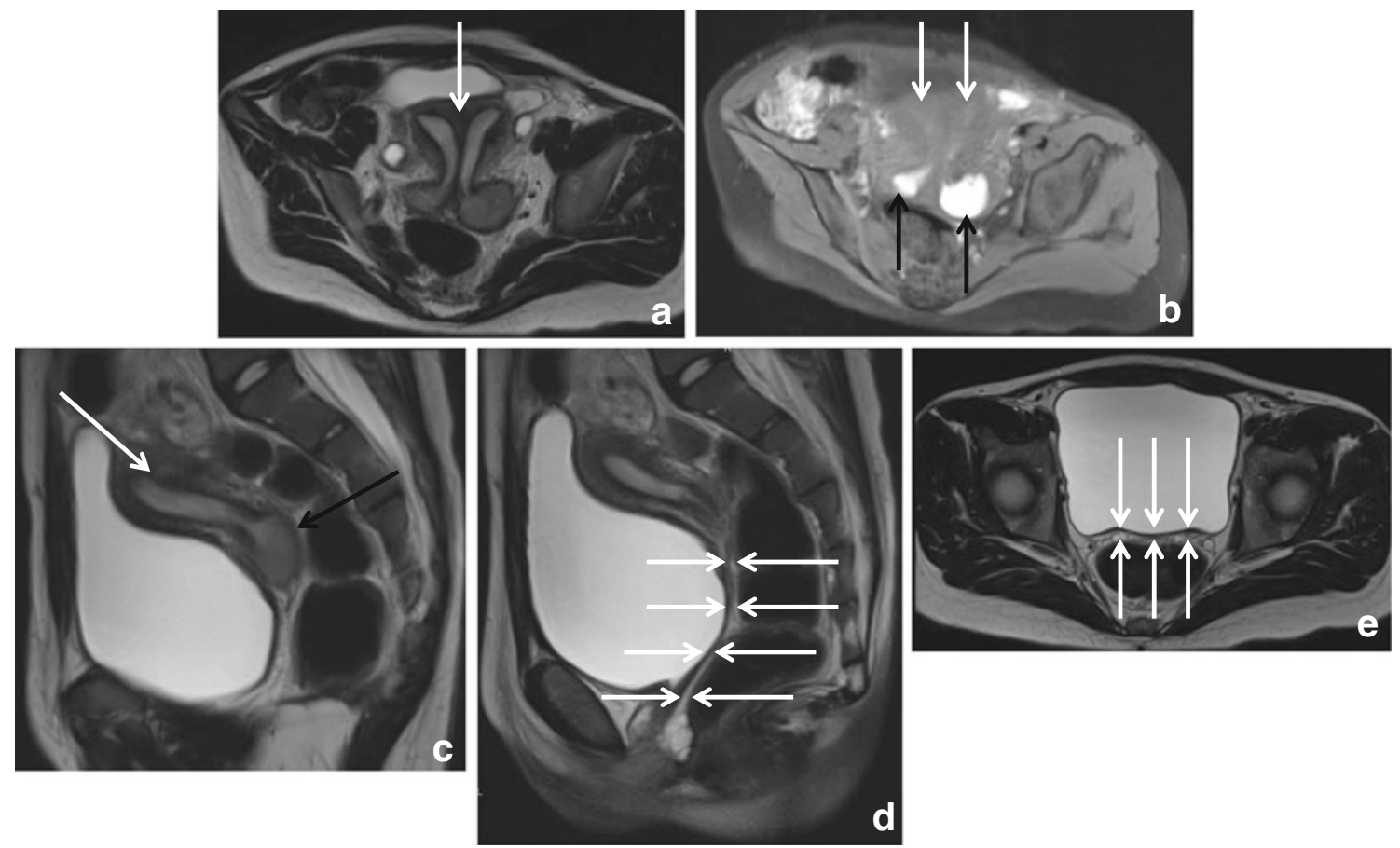

Fig. 1 Preoperative MRI views. a Axial T2-weighted image showing complete uterocervical septum (white arrow). b Axial T1-weighted image with hyperintensity corresponding to blood: hematometra (white arrow) and hematocervix (black arrow). c Sagittal T2weighted image showing one side of the malformation with hematometra

Because vaginal agenesis was complete, the vesicorectal space was dissected through the vaginal dimple and the pelvic peritoneum was opened following dissection of the retroperitoneal tissue. The cervix was reached and, after evacuation of the chocolate fluid, resection of the cervical septum was performed using cold scissors, with partial resection of the uterine septum due to the small size of the uterus. The cervix was sutured directly to the vaginal dimple using separate stitches (Vicryl 2-0 Johnson \& Johnson, Hamburg, Germany). A Foley catheter was introduced into the uterus to allow uterine permeability. Prophylactic anti-

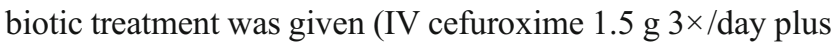
metronidazole IV $1.5 \mathrm{~g} 1 \times /$ day) for 2 days, followed by oral treatment. (white arrow) and hematocervix (black arrow). d Sagittal T2-weighted image showing complete vaginal agenesis (between white arrows). e Axial T2-weighted image showing complete vaginal agenesis (between white arrows)

Two weeks after surgery, vaginal examination was performed under sedation. The vulvar scar was deemed satisfactory. The intrauterine catheter was replaced and left in situ for another week.

Thirty-four months after surgery, the patient presented with regular menses, and vaginal examination showed a vaginal length of $3 \mathrm{~cm}$. Pelvic MRI showed a heart-shaped uterus with persistence of partial uterine septum and a single cervix with no blood accumulation (Fig. 3a). Both ovaries looked normal. On T2-weighted axial (Fig. 3c) and sagittal (Fig. 3b) images, the presence of a vagina containing mucus in the posterior fornix could be observed. The patient had not yet had sexual intercourse at the time of writing of the manuscript.
Fig. 2 Laparoscopic views. a Single heart-shaped uterus (black arrow). b Endometriotic implants on the uterosacral ligaments (black arrow) and in the ovarian fossa on both sides (white arrows)
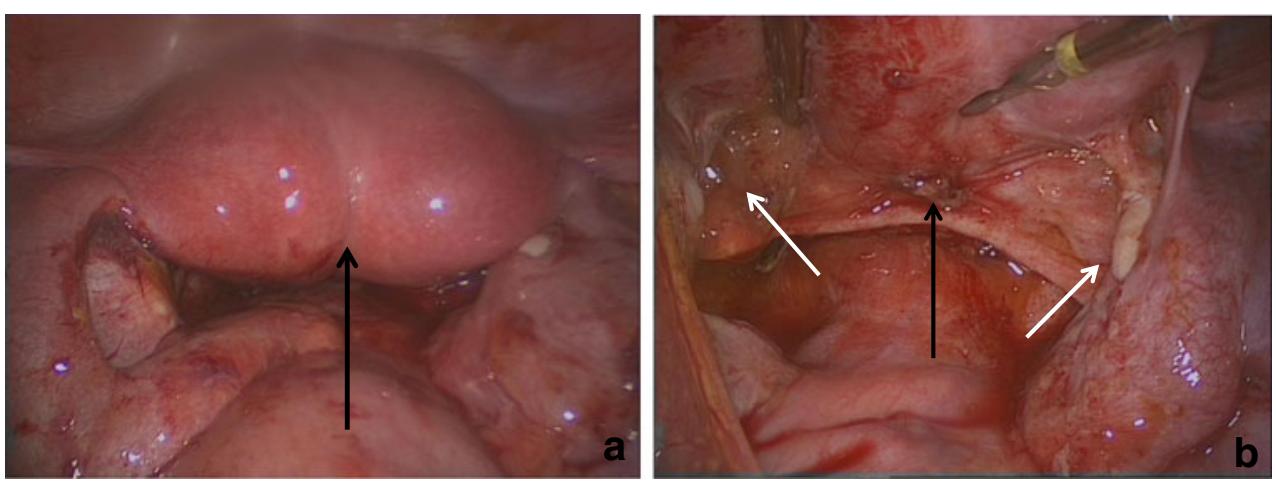
Fig. 3 Postoperative MRI T2weighted views. a Coronal T2weighted image showing a single cervix (white arrow) and heart-shaped uterus with partial uterine septum (black arrow). b Sagittal T2-weighted image showing the presence of a short vagina with a little mucus in the posterior fornix (between white arrows). c Axial T2-weighted image showing the presence of a vagina (between white arrows)

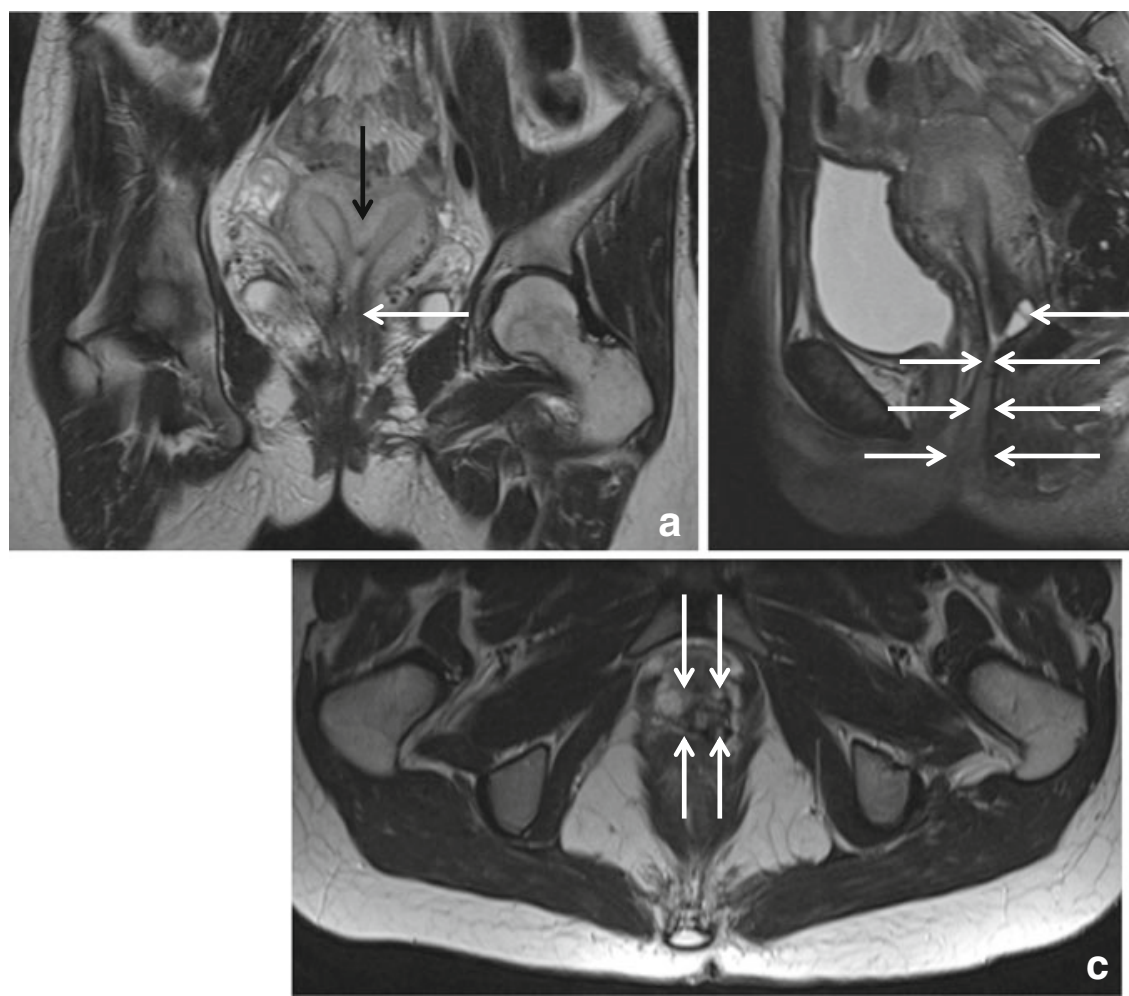

\section{Discussion}

Based on embryology, development of the female genital tract begins at 3 weeks of gestation and continues into the second trimester of pregnancy [4]. In females, gonadal development results from chromosomal factors acting on primordial germ cells in the genital ridge [4]. The genital tract develops independently of the gonads [4] and results from formation and fusion of the Müllerian ducts, urogenital sinus, and vaginal plate [5].

The cephalic portion of the Müllerian ducts develops into the fallopian tubes and the caudal portions fuse to form the uterus, cervix [6], and upper vagina [4]. The urogenital sinus develops into the two sinovaginal bulbs that proliferate into the caudal portion of the Müllerian ducts, forming the vaginal plate [4]. It appears that between 13 and 20 weeks, the septum formed by the median wall between the two Müllerian ducts undergoes resorption. Two theories may explain this resorption. It could be unidirectional from the caudal to the cranial part of the septum, or bidirectional originating in the isthmic portion and simultaneously extending caudally and cranially [7]. Degeneration of the central cells of the vaginal plate forms the lumen of the lower vagina and hymen [4]. Disruption at any point in this differentiation process can result in different types of congenital anomalies of the female genital tract [5].

In our case, fusion between the caudal portions of the Müllerian ducts was complete but resorption was not, causing septation of the uterus and cervix. The upper vagina was absent, probably due to the lack of fusion between the caudal portions of the Müllerian ducts and the urogenital sinus.

Genital malformations can have variable presentations, and different classifications have been made over the years. In 1988, the American Fertility Society proposed a simple classification system based on the anatomy of the female genital tract (especially the uterus) [2]. In 2011, Acién et al. put forward a revised version of an earlier clinical and embryological classification of genital malformations from 2004 [8]. In 2005, Oppelt et al. presented another classification based on the vagina cervix uterus adnexa-associated malformation. They added adnexal and extragenital anomalies that may be associated (renal, skeletal, cardiac, neurologic, and others) [9].

Most cases of vaginal agenesis encountered in the literature are MRKH syndrome, lower vaginal agenesis, or those associated with cervical atresia [10-14]. Oppelt et al. reported a cohort of 284 women with MRKH syndrome, only $0.7 \%$ of whom presented with a hypoplastic uterus. None of them had a normal-sized septate uterus [15].

In cases where vaginal agenesis is associated with a normal uterus, different surgical treatments have been proposed to create a neovagina and communication with the uterus. Darwish et al. [10] performed a technique of transretropubic balloon vaginoplasty in four patients. Only one patient had a functioning uterus and a second operation was required to achieve communication 
between the cervix and neovagina $[10,11]$. El Saman et al. [12] reported five cases of cervical atresia, two of which were associated with vaginal agenesis. They performed canalization of the cervical atresia by laparoscopy. A drain was passed through the uterine fundus, atretic cervix, and vagina or vaginal dimple (in case of associated vaginal agenesis). The drain was removed after the next menses. All patients presented with normal cycles, but their follow-up was short (less than 10 months). Kansagra et al. [13] performed ultrasoundguided balloon vaginoplasty in two patients to treat lower vaginal agenesis and complete transverse vaginal septum. After balloon dilatation of the vaginal agenesis/transverse septum, a Foley catheter was introduced into the upper vagina and left in place for 6 weeks. A 1-cm-diameter tract was obtained and the patients had painless menstruation.

In our particular case, the patient presented with complete vaginal agenesis and a septate uterus. The vaginal procedure assisted by laparoscopy allowed suture of the cervix directly to the vaginal dimple and resection of the uterocervical septum. The young patient subsequently experienced regular menses and the last follow-up was 34 months after surgery.

\section{Conclusion}

To our knowledge, this is the first case of uterocervical septum associated with complete vaginal agenesis to be described in the literature. The laparoscopy-assisted vaginal procedure allowed dissection of the vesicorectal space, creation of a 3-cm-length vagina, and establishment of regular menses. Further follow-up is now needed to evaluate the possibility of sexual intercourse after implementing this type of repair.

Conflict of interest The authors report no conflict of interest. The authors alone are responsible for the content and writing of the paper.

\section{References}

1. Capito C, Echaieb A, Lortat-Jacob S, Thibaud E, Sarnacki S, Nihoul-Fékété C (2008) Pitfalls in the diagnosis and management of obstructive uterovaginal duplication: a series of 32 cases. Pediatrics 122(4):e891-e897

2. Grimbizis GF, Campo R (2010) Congenital malformations of the female genital tract: the need for a new classification system. Fertil Steril 94(2):401-407

3. Creatsas G, Deligeoroglou E (2010) Vaginal aplasia and reconstruction. Best Pract Res Clin Obstet Gynaecol 24(2):185-191

4. Laufer MR (2011) Diagnosis and management of congenital anomalies of the vagina. Available at http://www.uptodate.com/ home/index.html.

5. Wright KN, Okpala O, Laufer MR (2011) Obstructed uteri with a cervix and vagina. Fertil Steril 95(1):290.e17-290.e19

6. Griffin JE, Wilson JD (2011) Normal sexual differentiation. Available at http://www.uptodate.com/home/index.html.

7. Ergün A, Pabuccu R, Atay V, Kücük T, Duru NK, Güngör S (1997) Three sisters with septate uteri: another reference to bidirectional theory. Hum Reprod 12(1):140-142

8. Acién P, Acién MI (2011) The history of female genital tract malformation classifications and proposal of an updated system. Hum Reprod Update 17(5):693-705, Review

9. Oppelt P, Renner SP, Brucker S, Strissel PL, Strick R, Oppelt PG, Doerr HG, Schott GE, Hucke J, Wallwiener D, Beckmann MW (2005) The VCUAM (vagina cervix uterus adnex-associated malformation) classification: a new classification for genital malformations. Fertil Steril 84(5):1493-1497

10. Darwish AM, Mohammad AM (2011) A new era for treating vaginal aplasia using transretropubic balloon vaginoplasty approach. Fertil Steril 95(3):1104-1108

11. El Saman AM (2010) Retropubic balloon vaginoplasty for management of Mayer-Rokitansky-Küster-Hauser syndrome. Fertil Steril 93(6):2016-2019

12. El Saman AM (2010) Endoscopically monitored canalization for treatment of congenital cervical atresia: the least invasive approach. Fertil Steril 94(1):313-316

13. Kansagra AP, Miller CB, Roberts AC (2011) A novel image-guided balloon vaginoplasty method to treat obstructive vaginal anomalies. $\mathrm{J}$ Vasc Interv Radiol 22(5):691-694

14. Growdon WB, Laufer MR (2008) Uterine didelphys with duplicated upper vagina and bilateral lower vaginal agenesis: a novel Müllerian anomaly with options for surgical management. Fertil Steril 89(3):693-698

15. Oppelt PG, Lermann J, Strick R, Dittrich R, Strissel P, Rettig I, Schulze C, Renner SP, Beckmann MW, Brucker S, Rall K, Mueller A (2012) Malformations in a cohort of 284 women with MayerRokitansky-Küster-Hauser syndrome (MRKH). Reprod Biol Endocrinol 10(1):57 